\title{
Introduction to the teaching course in surgical oncology
}

Nowadays, surgery still remains the most efficient treatment, and sometimes the only one, for a great number of malignancies, some of them highly prevalent. However, the addition of neoadjuvant or adjuvant treatments to surgery such as radiotherapy or chemotherapy has been proved to continuously increase survival or diminish morbidity in some of these neoplasias. Therefore, multidisciplinary approaches are of paramount importance in the management of many cancers.

Actually, surgical oncology is not established as a discipline that needs specific competence in our country. However, the progress in the knowledge of the molecular features that determine the behaviour in diffe- rent tumors will affect our making decision in the future. In addition, the increasing effectiveness of multidisciplinary approaches along with the use of sophisticated surgical techniques and strategies among others strengthen the need of specific training. In this issue, we begin in the Sección Docente/Teaching Section of Revista de Oncología a series of topics selected by the Sociedad Española de Oncología Quirúrgica (SEOQ). The first of them deals with the selective biopsy of sentinel node that has recently dispelled the body of controversy maintained along decades on indications of radical lymphadenoctomy in some tumors such as melanoma. 